\title{
DEVELOPMENT OF DIRECT-DETECTION DOPPLER WIND LIDAR FOR VERTICAL ATMOSPHERIC MOTION
}

\author{
Shoken Ishii ${ }^{12^{*}}$, Makoto Aoki ${ }^{1}$, Kanna Tominaga ${ }^{2}$, Tomoaki Nishizawa ${ }^{3}$, Yoshitaka Jin ${ }^{3}$, \\ Nobuo Sugimoto $^{3}$, Yasukuni Shibata ${ }^{2}$, Atsushi Sato ${ }^{4,1}$, Kaori Sato ${ }^{5}$, and Hajime Okamoto \\ ${ }^{I}$ National Institute of Information and Communications Technology, Koganei Tokyo 184-8795, Japan \\ ${ }^{2}$ Tokyo Metropolitan University, Hino Tokyo 191-0065, Japan \\ ${ }^{3}$ National Institute of Environmental Studies, Tsukuba Ibaraki 305-8506, Japan \\ ${ }^{4}$ Tohoku Institute of Technology, Sendai Miyagi 982-8577, Japan \\ ${ }^{5}$ Kyushu University, Kasuga Fukuoka 816-8580, Japan \\ *Email: sishii@nict.go.jp
}

\begin{abstract}
Wind is fundamental in many atmospheric phenomena. Global wind profile observation is important to improve numerical weather prediction (NWP) and various meteorological studies. Wind profile observations are measured mainly by radiosonde networks. A Doppler Wind Lidar (DWL) is a useful remote sensing technique for wind measurement. DWL would provide us with a wind profile having high vertical resolution, low bias, and good precision. The National Institute of Information and Communications Technology (NICT) studies DWL has been developing various DWL. In the paper, we report development of a 355-nm direct-detection DWL and describe recent results of a $2-\mu \mathrm{m}$ coherent DWL at NICT.
\end{abstract}

\section{INTRODUCTION}

Current space-based passive observing systems for wind measurement is limited [1]. The current space-based passive observing systems have a large coverage area and high temporal and horizontal resolutions but has a low vertical resolution. The World Meteorological Organization (WMO) wants to develop spacebased wind profiling systems [2]. A Doppler Wind Lidar (DWL) can provide us with a wind profile with high vertical resolution, low bias, and good precision. A space-based DWL can fill the gap for the current space-based passive observing systems. The European Space Agency (ESA) launched a first space-based DWL called 'Aeolus' for the global wind profile observation in 2018 [3]. The National Institute of Information and Communications Technology (NICT) is studying the feasibility to realize the future space-based DWL [4-6]. NICT developed a ground-based 2- $\mu \mathrm{m}$ coherent DWL for $\mathrm{CO}_{2}$ and wind measurements [7]. We have a plan to make wind measurement around and inside clouds with 355$\mathrm{nm}$ direct-detection and $2-\mu \mathrm{m}$ coherent DWL measurements. We are developing a ground-based direct-detection DWL. In the paper, we describe development and progress of the two DWLs.

\section{DEVELOPMENT of 355-NM DIRECT- DETECTION DOPPLER WIND LIDAR}

\subsection{5-nm direct-detection lidar system}

A direct-detection DWL has the advantage that wind measurement can be made any aerosol conditions because it uses the light backscattered by molecules (Rayleigh scattering). This feature is one of the characteristics of the direct-detection DWL. NICT is developing a direct-detection DWL with a double-edge technique. Figure 1 and Table 1 show a block diagram and specifications of the direct-detection DWL. The direct-detection DWL uses a flash-lump-pumped Q-switched Nd:YAG laser (Contuinum: Surelite I), an o SchmidtCassegrain type (Celestron: C14), a Fabry-Perot etalon, a knife-edge right-angle prism mirror, and two photomultiplier tubes (Hamamatsu: R9880U113), The single-frequency a flash-lump-pumped Q-switched Nd:YAG operating at a wavelength of $0.355 \mu \mathrm{m}$ can emit an output energy of $100 \mathrm{~mJ}$ with a pulse width of $10 \mathrm{~ns}$ (FWHM) at a pulse repetition frequency of $10 \mathrm{~Hz}$. The wavelength of backscattered light is Doppler-shifted from that of the incident laser light by the moving molecules and aerosols. The backscattered light is collected by a telescope and split into two paths using a wedge plate. Each split light beam passes through a Fabry-Perot étalon (which functions as an edge filter) and is focused on each detector. The number 
of photons, $\mathrm{N}_{\mathrm{i}=1,2}$ detected at each detector for a line-of-sight (LOS) wind speed of $v_{L O S}(\mathrm{~m} / \mathrm{s})$ is given as Eq. (1),
Since the photon noise is the dominant noise source, we assume that the standard deviation of $\mathrm{N}_{\mathrm{i}}$ is given by a Poisson distribution. $\mathrm{SNR}_{\mathrm{i}}$ is given as Eq. (2),

$$
\begin{aligned}
N_{s, i}\left(v_{L O S}\right) & =T_{B S} \cdot \frac{E_{T} \cdot F r e q \cdot \Delta t}{h v_{L}} \cdot A_{T} \cdot T_{0} \cdot O_{A}(r) \cdot Q_{e} \cdot \frac{\Delta r}{r^{2}} \cdot T_{A}{ }^{2} \\
& \times\left[\beta_{M}(r) \cdot \int_{-\infty}^{\infty} T_{F, i}(v) \cdot\left\{G_{L} * G_{M}(v, \Delta v)\right\} d v+\beta_{\Lambda}(r) \cdot \int_{-\infty}^{\infty} T_{F, i}(v) \cdot\left\{G_{L} * L_{A}(v, \Delta v)\right\} d v\right],
\end{aligned}
$$

where $\mathrm{T}_{\mathrm{BS}}$ is the beam-splitter transmittance, $\mathrm{E}_{\mathrm{T}}$ is the transmitted laser energy, Freq is a pulse repetition frequency, $\Delta \mathrm{t}$ is integration time, $v_{\mathrm{L}}$ is the laser frequency, $A_{T}$ is the telescope area, $T_{o}$ is optical efficiency of the system without etalon transmission, $\mathrm{O}_{\mathrm{A}}(\mathrm{r})$ is an overlap function, $\mathrm{Q}_{\mathrm{e}}$ is quantum efficiency of the detector, $\Delta r$ is range bin length, $r$ is a distance to the target atmosphere, $T_{A}$ is atmospheric transmission, $\mathrm{T}_{\mathrm{F}, \mathrm{i}=1,2}(\mathrm{v})$ is transmission at a frequency of $v$ for an edge filter centered at $\nu_{\mathrm{L}}, \beta_{\mathrm{m}}$ and $\beta_{\mathrm{a}}$ are the molecule and aerosol backscattering coefficients, $\mathrm{G}_{\mathrm{L}}$ and $\mathrm{G}_{\mathrm{m}}$ are Gaussian line-broadening functions for the laserline spectrum and the Rayleigh-Brillouin spectrum, $\mathrm{L}_{\mathrm{a}}$ is a Lorentzian function for the aerosol-line spectrum, $\Delta v$ is the Doppler-shifted frequency, the asterisk denotes a convolution operation, ' $h$ ' is Planck's constant, and 'c' is the light speed.

$$
S N R_{i}=\sqrt{\text { Freq } \cdot \Delta t} \cdot \frac{N_{i} / \text { Freq } \cdot \Delta t}{\sqrt{N_{i} / \text { Freq } \cdot \Delta t+N_{D C}+N_{B G}}},
$$

where $\mathrm{N}_{\mathrm{DC}}$ and $\mathrm{N}_{\mathrm{BG}}$ are the dark current noise of the detector and the background noise. The location of the $i$ th edge filter is determined by the following condition described by Flesia and Korb [8]. the LOS wind speed error $\sigma_{\text {LOS }}$ is given by

$$
\sigma_{L O S}=\frac{c}{\frac{2}{v} \cdot\left(\frac{1}{N_{1}(0)} \frac{d}{d v} N_{S, M, 1}(0)+\frac{-1}{N_{2}(0)} \frac{d}{d v} N_{S, M, 2}(0)\right)} \cdot \sqrt{\frac{1}{S N R_{1}^{2}}+\frac{1}{S N R_{2}^{2}}}
$$

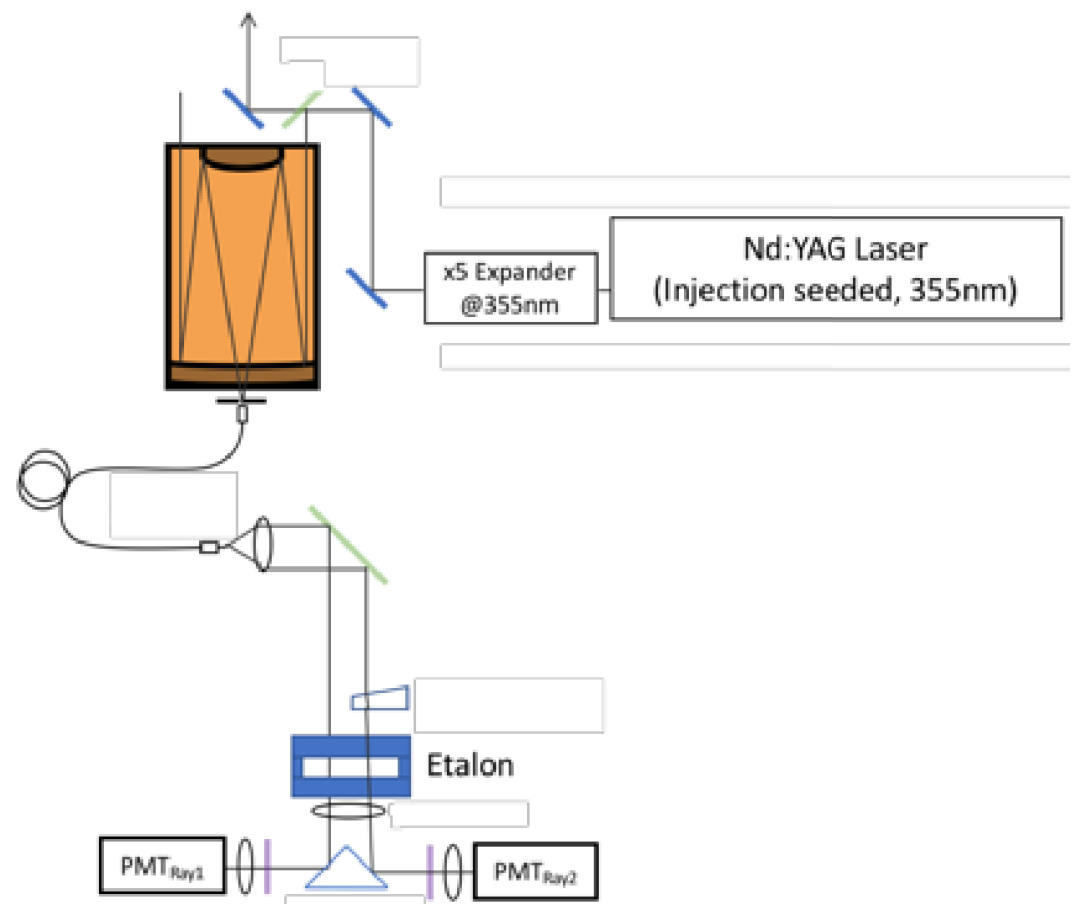

Figure 1. Block diagram of 355-nm direct-detection Doppler wind lidar. 
Table 1. Specifications of 355-nm direct-detection Doppler wind lidar.

\begin{tabular}{|cl}
\hline Transmitter & \\
\hline Laser & Nd:YAG, \\
Wavelength & $355 \mathrm{~nm}$ \\
Pulse energy & $100 \mathrm{~mJ}$ \\
Pulse width & $10 \mathrm{nsec}$ \\
PRF & $10 \mathrm{~Hz}$ \\
Beam divergence & $0.2 \mathrm{mrad}$ \\
\hline Receiver & \\
\hline Telescope & Schmidt-Cassegrain \\
Diameter & $280 / 350 \mathrm{~mm}$ \\
FOV & $0.1 \mathrm{mrad}$ \\
Separators & Fabry-Perot etalon \\
Detector & PMT \\
\hline Data acquisition & \\
\hline Signal processing & $11 \mathrm{Bit} \mathrm{A} / \mathrm{D}$ \\
Sampling frequency & $40 \mathrm{MHz}$ \\
Sampling points & 65536 \\
\hline
\end{tabular}

\subsection{Wind Measurement performance of $355 \mathrm{~nm}$ direct-detection DWL}

Figure 2 shows the profile of the LOS wind error calculated from Eqs (1) through (3) for a LOS wind speed of $1 \mathrm{~m} / \mathrm{s}$ for the various accumulation laser pulse: $100,600,3000$, and 6000 . The figure shows that altitudes for the LOS wind speed errors of 0.5 $\mathrm{m} / \mathrm{s}$ for the four accumulation laser pulses are 2, 5, 10 , and $15 \mathrm{~km}$. Our coherent lidar developed for and wind measurements can make vertical wind speed measurement at altitudes up to about $10 \mathrm{~km}$ for the accumulation laser pulse of 30 corresponding to $1 \mathrm{sec}$, where depends on the load of aerosol or clouds. When SNR is high, the $2-\mu \mathrm{m}$ coherent lidar can make wind measurement with random error of 0.12 $\mathrm{m} / \mathrm{sec}$ and vert low bias of $<0.1 \mathrm{~m} / \mathrm{sec}$ under the real atmospheric conditions [9]. The appropriate accumulation laser pulses for the dual DWL measurements are 100 and 600 shots.

\section{VERTICAL WIND MEASUREMENT PERFORMANCE OF $2-\mu M$ COHERENT DOPPLER WIND LIDAR}

A ground-based 2- $\mu \mathrm{m}$ coherent DWL was developed at NICT in 2013. The block diagram specifications of the ground-based coherent DWL are shown in Figure 3 and Table 2, respectively. The ground-based DWL uses a conductively-cooled laser-diode-pumped Q-switched Tm,Ho:YLF laser,

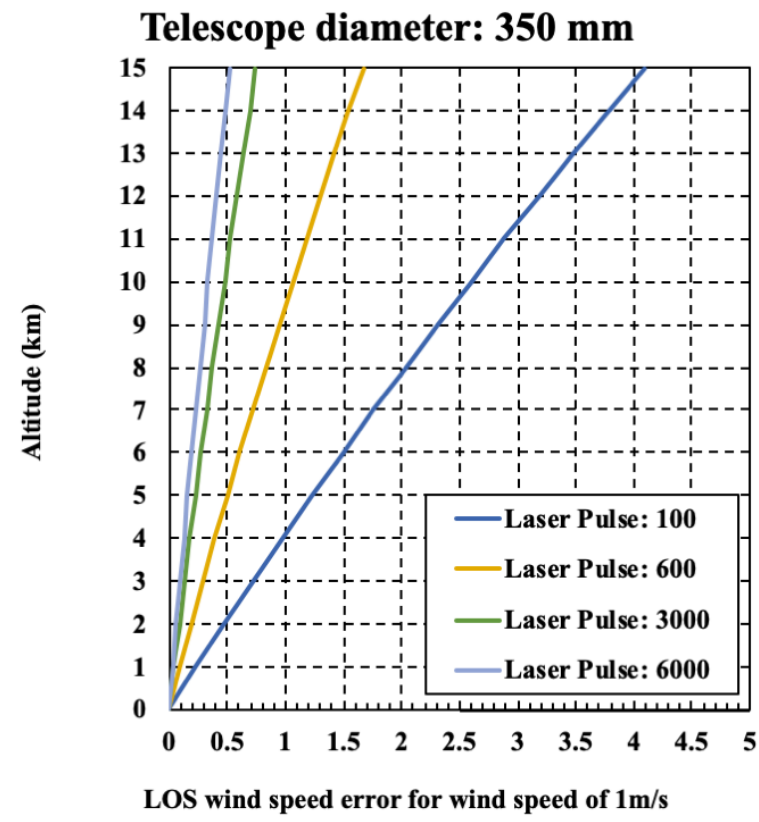

Figure 2. Profiles of the LOS wind speed error for various accumulation laser pulse:100, 600, 3000, and 6000 .

an off-axis telescope, two heterodyne detectors, and signal processing devices. The singlefrequency Q-switched Tm,Ho:YLF laser an emit an output energy of $90 \mathrm{~mJ}$ with a pulse width of $150 \mathrm{~ns}$ (FWHM) at a pulse repetition frequency of $40 \mathrm{~Hz}$. The bias and error in the LOS wind speed measurement were also investigated by returns backscattered from a building (hard target) located at about $25 \mathrm{~km}$. The ground-based coherent DWL can make the LOS wind speed with a low bias of $<0.0 \mathrm{~m} / \mathrm{sec}$ and random error of about $0.1 \mathrm{~m} / \mathrm{sec}$ estimated from 10-shot averaged zero LOS wind speeds. Figure 4 shows the vertical wind speed measurements of atmosphere, aerosols and clouds by the ground-based coherent DWL at NICT headquarters on 27 March 2018. The ground-based coherent DWL detected details of thermal convections near in the atmospheric boundary layer.

\section{SUMMARY}

A DWL is a useful remote sensing technique for wind measurement. DWL would provide us with a wind profile with high vertical resolution, low bias, and good precision. We have plan to make vertical wind measurement around and in side clouds with 


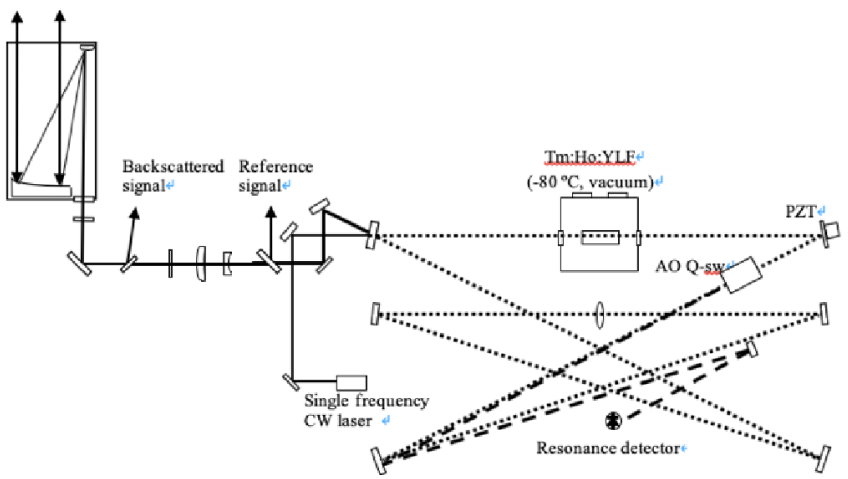

Figure 3. Block diagrams of 2- $\mu \mathrm{m}$ coherent Doppler wind lidar.

Table 2. Specifications of 2- $\mu \mathrm{m}$ coherent Doppler wind lidar.

\begin{tabular}{ll}
\hline Transmitter & \\
Laser & Tm,Ho:YLF \\
& $2051.002-2051.058 \mathrm{~nm}$ (On) \\
Wavelength & $2051.250 \mathrm{~nm}$ (Off) \\
Pulse energy & $50-80 \mathrm{~mJ} /$ pulse (Target) \\
Pulse width & $150 \mathrm{~ns}$ (FWHM) \\
Pulse repetition & $>30 \mathrm{~Hz}$ \\
Polarization & Circular \\
Receiver & \\
Telescope type & Mersenne off-axis \\
Diameter & $10 \mathrm{~cm}$ \\
Magnification & $10 \mathrm{x}$ \\
Detector & InGaAs-PIN photodiode \\
DET & Balanced InGaAs-PIN photodiode \\
Resolution & $14-$ bit A/D conversion \\
Sampling frequency & $400 \mathrm{MHz}$ \\
\hline
\end{tabular}
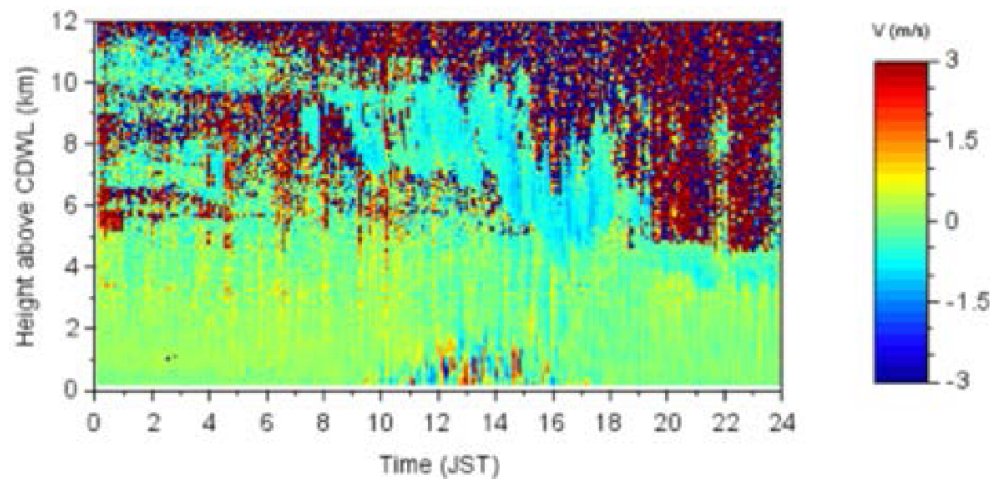

Figure 4. Temporal variation of vertical wind speed and SNR observed by $2-\mu \mathrm{m}$ coherent DWL on 27 Mach, 2018.

direct-detection and coherent DWL wind measurements. NICT is a developing a directdetection DWL with the double-edge technique. Our result shows that the appropriate accumulation laser pulses of 100 and 600 shots is appropriate for the direct-detection DWL wind measurement. The $2-\mu \mathrm{m}$ coherent DWL can describe temporal variation of atmosphere. The combination of direct-detection and coherent DWL wind measurement would be useful for investigating details of various motions in the atmosphere. The dual DWL wind measurements with a cloud radar measurement. would be suitable to evaluate and characterize vertical wind motion around and inside clouds.

\section{ACKNOWLEDGEMENTS}

This research is supported by JSPS KAKENHI under Grant Numbers JP17H06139. A part of this research was supported by the Collaborated Research Program of Research Institute for Applied Mechanics, Kyushu University.

\section{REFERENCES}

[1] W. E. Baker et al. Bull. Am. Meteorol. Soc., 95, 543564 (2014).

[2] WMO. WMO Tech. Rep. 2012-1, 6 (2012).

[3] ESA. SP-1233(4), (1999).

[4] S. Ishii et al. J. Meteorol. Soc. Jpn.95, 301-317 (2017).

[5] P. Baron et al. J. Meteorol. Soc. Jpn 95, 319-342 (2017).

[6] K. Okamoto et al. J. Meteorol. Soc. Jpn. 96, 179-199 (2018).

[7] S. Ishii et al. Appl. Opt., 49, 1809-1817 (2010).

[8] Flesia and Korb, Appl. Opt. 38, 432-440 (1999).

[9] Iwai et al. J. Atmos. Ocean. Technol., 30(3), 429-449 (2013). 